\title{
Capture by hybridization for full-length barcode-based eukaryotic and prokaryotic biodiversity inventories of deep sea ecosystems
}

\author{
Babett Günther $^{1}$ (1) | Sophie Marre ${ }^{2}$ | Clémence Defois $^{2}$ | Thomas Merzi ${ }^{3}$ | \\ Philippe Blanc $^{3}$ | Pierre Peyret ${ }^{2}$ | Sophie Arnaud-Haond ${ }^{1}$
}

${ }^{1}$ MARBEC, Universite of Montpellier, CNRS, Ifremer, IRD, Sète, France

${ }^{2}$ Université Clermont Auvergne, INRAE, UMR 0454 MEDIS, Clermont-Ferrand, France

${ }^{3}$ Total SE, Centre Scientifique et Technique Jean Feger, Pau, France

\section{Correspondence}

Babett Günther and Sophie ArnaudHaond, MARBEC, University of Montpellier, CNRS, Ifremer, IRD, Sète, France.

Emails: guenther.babett@gmail.com; sarnaud@ifremer.fr

Funding information Total, Grant/Award Number: Contract no. 9194 TOTAL FRO000; European Union 2020 Research and Innovation Programme, Grant/Award Number: 678760

\begin{abstract}
Biodiversity inventory of marine systems remains limited due to unbalanced access to the three ocean dimensions. The use of environmental DNA (eDNA) for metabarcoding allows fast and effective biodiversity inventory and is forecast as a future biodiversity research and biomonitoring tool. However, in poorly understood ecosystems, eDNA results remain difficult to interpret due to large gaps in reference databases and PCR bias limiting the detection of some major phyla. Here, we aimed to circumvent these limitations by avoiding PCR and recollecting larger DNA fragments to improve assignment of detected taxa through phylogenetic reconstruction. We applied capture by hybridization ( $\mathrm{CBH}$ ) to enrich DNA from deep-sea sediment samples and compared the results with those obtained through an up-to-date metabarcoding PCRbased approach (MTB). Originally developed for bacterial communities and targeting $16 \mathrm{~S}$ rDNA, the $\mathrm{CBH}$ approach was applied to $18 \mathrm{~S}$ rDNA to improve the detection of species forming benthic communities of eukaryotes, with a particular focus on metazoans. The results confirmed the possibility of extending $\mathrm{CBH}$ to metazoans with two major advantages: (i) $\mathrm{CBH}$ revealed a broader spectrum of prokaryotic, eukaryotic, and particularly metazoan diversity, and (ii) $\mathrm{CBH}$ allowed much more robust phylogenetic reconstructions of full-length barcodes with up to 1900 base pairs. This is particularly important for taxa whose assignment is hampered by gaps in reference databases. This study provides a database and probes to apply $18 \mathrm{~S} \mathrm{CBH}$ to diverse marine systems, confirming this promising new tool to improve biodiversity assessments in data-poor ecosystems such as those in the deep sea.

KEYWORDS

16S, 18S, environmental DNA, metabarcoding, metazoan
\end{abstract}

\section{1 | INTRODUCTION}

Over two-thirds of the Earth is covered by oceans, probably sheltering a high level of still poorly studied biodiversity, particularly in the deep sea (Costello \& Chaudhary, 2017; Costello et al., 2010). Today, molecular approaches provide nonintrusive methods to study the diversity of marine environments, even those that are hardly accessible to sampling. The analysis of environmental DNA (eDNA; Taberlet et al., 2012) represents a promising path to inventory biodiversity and sets the ground for the development of molecular biomonitoring protocols (Andruszkiewicz et al., 2017; Apothéloz-PerretGentil et al., 2017; Bohan et al., 2017; Cordier et al., 2018; Derocles 
et al., 2018). Approaches based on eDNA (air, ground, sediment, or water, relatively easy to access and sample) target the genetic material present in the environment (Bohmann et al., 2014; Thomsen \& Willerslev, 2015), allowing us to unravel the nature of macro- and micro-organisms present in the surrounding habitats. First developed for the uncultivable majority that represents the microbial world (Xu, 2006), metabarcoding approaches, relying on PCR-based amplicon sequence identification combined with high-throughput sequencing, were transferred to eukaryotes early on (Creer et al., 2010; Hajibabaei et al., 2011; Taberlet et al., 2012; Valentini et al., 2009).

Over the last decade, PCR-based metabarcoding protocols have been improved, from sampling up to bioinformatic steps, to optimize their resolution and interpretation. Nevertheless, biomonitoring and biodiversity inventory using metabarcoding are challenging (Miya et al., 2015; Yamamoto et al., 2017) for two main reasons. First, this method relies on PCR-based DNA enrichment, suffering biases due to unequal amplification across taxa (PCR bias) and artefacts (PCR errors prominent to sequence errors), leading to biased biodiversity inventories (Acinas et al., 2005; Kanagawa, 2003; Sefc et al., 2007; Smyth et al., 2010). Second, the evolution of high-throughput sequencing technologies available on the market led to higher yield but shorter sequencing fragments, limiting the use of metabarcoding to short fragments. Such short fragments (usually 150 to 450 base pairs) lead to a less reliable assignment of sequences to taxa and hamper the use of data produced for comprehensive phylogenetic reconstructions. This is particularly limiting in ecosystems where biodiversity is poorly described and reference databases contain large gaps, for which many unassigned sequences can correspond to existing undescribed biodiversity, yet teasing them apart from spurious sequences would require phylogenetic reconstruction. The limiting factor for taxonomic assignment of deep sea organisms is the general lack of sequence references in marine systems. Some major groups, such as nematodes, which are the most abundant and diverse benthic metazoan taxa, can rarely be identified genetically (Dell'Anno et al., 2015; Gambi \& Danovaro, 2016). Thus, long, high-quality barcode libraries are needed to improve taxonomic identification in general, especially for poorly known groups.

Theoretically, direct metagenomic sequencing (such as shotgun sequencing) could solve these limitations, as these sequences can also be reconstructed from eDNA to obtain a comprehensive overview of the taxonomic diversity of the studied community, free of PCR bias (Porter \& Hajibabaei, 2018) and allowing reliable phylogenies based on long fragments. However, the production of metagenomes is still extremely costly, leading to a dominance of prokaryotic sequences, and the de novo reconstruction of comprehensive metagenomes is highly time consuming; differentiating between biological differences and sequencing errors is hardly possible and highly limited by gaps in reference databases (Ghurye et al., 2016; Quince et al., 2017).

As an intermediate, less expensive option, to avoid the two main limitations associated with PCR-based metabarcoding, two other methods of DNA enrichment are available (Mamanova et al., 2010; Mertes et al., 2011): the molecular inversion probe (MIP) and capture by hybridization ( $\mathrm{CBH}$ ). $\mathrm{CBH}$ exists in two variations,"onarray capture" on a solid microarray or "in-solution capture", which takes place within a fluid medium (Gasc et al., 2016). Here, we use the latter, which was first described by Gnirke et al. (2009) for human exome resequencing, whereby hybrid probes are designed to enrich genomic DNA. While the initial cost of this system is high, by multiplexing libraries, efficient sequencing of several samples (up to 96-well plates) has been shown to be highly efficient (Meyer \& Kircher, 2010). Moreover, a diversity of probes (singlestranded sequences of DNA) designed in different locations of the target gene regions allows capturing a much wider diversity and recovering long fragments, thereby improving taxonomic assignment and allowing reasonable phylogenetic reconstruction (Denonfoux et al., 2013; Gasc \& Peyret, 2018). Furthermore, a low concentration DNA template is sufficient, allowing this method to be successfully used in low biomass environments (such as air or deep-sea biomes) wherein generally lower DNA concentrations are obtained, as in deep oligotrophic aquifers (Ranchou-Peyruse et al., 2017). It was also used to increase the relative abundance of ancient eDNA by up to 9-fold in an analysis of eukaryotes in marine sediment (Armbrecht et al., 2021). The first test using eDNA showed that a 100-fold lower concentration can be detected with $\mathrm{CBH}$ than with traditional methods (Seeber et al., 2019), while others mentioned reduced tractability for DNA with less than $0.1 \mathrm{ng}$ of total gDNA (Wilcox et al., 2018). Testing within complex prokaryotic communities even allowed the detection of extremely rarely represented members (less than 0.0001\%) (Gasc \& Peyret, 2018). It has been suggested that the final success of this method depends strongly on the probes (Ribière et al., 2016) rather than on the initial biomass and DNA concentration.

Improved biodiversity assessments can thus be expected using $\mathrm{CBH}$, (i) avoiding PCR steps, yet targeting a broader range of biodiversity in a single reaction by using a comprehensive and versatile set of probes and (ii) reconstructing long fragments for full barcode regions, allowing reliable phylogenetic positioning and reconstruction. In recent years, this methodology has proven to markedly improve microbial diversity inventories with precise taxonomic affiliation at the species level (Gasc \& Peyret, 2018). CBH was also applied to recover full-length microbial eukaryotic cDNAs in complex environmental samples (Bragalini et al., 2014) and to directly capture long DNA fragments (Gasc \& Peyret, 2017). Additionally, CBH using mitochondrial barcodes for inventories of metazoans in bulk or ethanol-preserved samples resulted in a highly accurate census of species (Gauthier et al., 2020; Shokralla et al., 2016), and similar results were obtained when testing the detection of a broad range of metazoans, including mammals, from aquatic and sediment eDNA samples (Seeber et al., 2019; Wilcox et al., 2018). Additionally, CBH represents a promising path for phylogenetic studies, as recently shown for butterflies (Kawahara et al., 2018).

With this study, we aimed to assess the potential of $16 \mathrm{~S}$ and $18 \mathrm{~S}$ rDNA enrichment by $\mathrm{CBH}$ coupled with high-throughput sequencing 
to explore the biodiversity of prokaryotes and eukaryotes, including metazoans, in the deep sea ( 500-2800 $\mathrm{m}$ depth). We analysed eDNA samples extracted from sediment to compare CBH with PCRbased metabarcoding for the V4 16S rDNA region and the V1-V2 $18 \mathrm{~S}$ rDNA region.

\section{2 | MATERIALS AND METHODS}

\section{1 | Samples}

The study included ten sediment samples from different deepsea areas (Appendix S1, Table 1), totaling five stations: four in the Atlantic Ocean and one in the Mediterranean Sea (see Figure 1, Table 1). Standardized sampling was performed using a Multicorer or Pushcorer (both with cores of $20 \mathrm{~cm}^{2}$ ) and slicing under sterile conditions. For this study, only the first two layers of sediment $(0-1 \mathrm{~cm}$ and $1-3 \mathrm{~cm})$ were used for each station. Samples were frozen at $-80^{\circ} \mathrm{C}$ immediately after recovery and slicing for later extraction. DNA extraction was performed using $10 \mathrm{~g}$ of substrate with the DNeasy PowerSoil Kit (Qiagen) following the manufacturer's protocols. Extraction controls were included (empty sampling bags conditioned onboard and rinsed with water and manufacturer tubes initially filled with sterile water instead of sediment). Final extracts were concentrated with three volumes $70 \%$ ethanol for precipitation reaction and diluted in molecular grade water.

\section{2 | Hybridization capture approach}

\subsubsection{Design of capture probes and in silico tests}

Probes were defined to target $16 \mathrm{~S}$ and $18 \mathrm{~S}$ rDNA genes to ensure specificity against the targeted genes while allowing the detection of sequence variants not yet described in databases by selecting

TAB LE 1 Samples, stations and positions displayed in Figure 1. Included are the station, depth of sediment from the core (slice), seafloor depth, and topographic features. Appendix S1 includes all further information on the samples and samplings

\begin{tabular}{lllll} 
Station & Nr. & $\begin{array}{l}\text { Slice in } \\
\mathrm{cm}\end{array}$ & $\begin{array}{l}\text { Depth } \\
\text { in } \mathrm{m}\end{array}$ & $\begin{array}{l}\text { Topographic } \\
\text { feature }\end{array}$ \\
\hline 1 & 1A & $0-1$ & 2683.5 & Hydrothermal vent \\
& 1B & $1-3$ & & \\
\hline 2 & 2A & $0-1$ & 2826 & Hydrothermal vent \\
\hline 3 & 2B & $1-3$ & & \\
\hline 4 & 3A & $0-1$ & 1325 & Seamount \\
\hline 5 & 3B & $1-3$ & & \\
\hline 5 & 4A & $0-1$ & 1920 & Seamount \\
& 4B & $1-3$ & & \\
\hline & 5A & $0-1$ & 554 & Seamount \\
\hline
\end{tabular}

degenerate probes (Table 2). Probes targeting specifically eukaryotes using 18S rDNA were designed using the same strategy as the previously published one for $16 \mathrm{~S}$ rDNA probes (Gasc \& Peyret, 2018). Briefly, probes were designed using KASpOD (Parisot et al., 2012) and PhylArray algorithms (Militon et al., 2007) and a custom curated database derived from all 18S rDNA sequences from the EMBL and similarly built for PhyloPDb development (Jaziri et al., 2014). Selection of probes distributed over the entire length of the gene and their length (31 to $50 \mathrm{bp}$ ) allow specific hybridization with their target even if mismatches are present. Every k-mer of 31- or 50-mer length was extracted from reference sequences. Then, fully overlapping k-mers were clustered at an $88 \%$ identity to produce a degenerate consensus sequence that takes into account sequence variability at each position (Parisot et al., 2012). Among the combinations derived from each degenerate probe, some correspond to sequences not previously included in public databases. They should, therefore, allow for the exploration of the as yet undescribed fraction of environmental microbial communities. Such strategies anticipate genetic variations and can thus detect both already known and still unknown sequences in environmental samples (Dugat-Bony et al., 2011). For each set of probes, coverage among SSU sequences from the SILVA database (release 132; Quast et al., 2013) was predicted by mapping probes

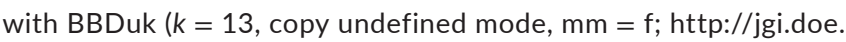
gov/data-and-tools/bb-tools/). Finally, adaptors containing the T7 promoters "ATCGCACCAGCGTGT" and "CACTGCGGCTCCTCA" were added to the $5^{\prime}$ and $3^{\prime}$ ends of probes, respectively, to enable PCR amplification (Ribière et al., 2016). These probes were designated capture probes.

\subsection{2 | Capture and sequencing}

Illumina libraries were constructed from eDNA samples using the Nextera XT (for nine samples) or TruSeq (for 1A) Kits (Illumina). Biotinylated RNA capture probes were obtained by in vitro transcription following the protocol described by Ribière et al. (2016). Hybridization capture, illustrated in Figure 2, was carried out independently for each sample with $16 \mathrm{~S}$ and $18 \mathrm{~S}$ rDNA probes at a ratio of 50/50, as described by Gasc and Peyret (2018). In brief, $500 \mathrm{ng}$ of sequencing library mixed with $2.5 \mu \mathrm{g}$ of salmon sperm DNA was incubated with 500 ng of biotinylated probes in a hybridization buffer $(10 \times$ SSPE, Denhardt's $10 \times$ solution, $10 \mathrm{mM}$ EDTA and $0.2 \% \mathrm{SDS}$ ) for $24 \mathrm{~h}$ at $65^{\circ} \mathrm{C}$. After hybridization, the probe/ target heteroduplexes were captured with $500 \mu \mathrm{g}$ of paramagnetic beads coated with streptavidin (Dynabeads M-280 Streptavidin, Invitrogen). Beads were collected using a magnetic stand (Ambion), washed once with $500 \mu \mathrm{l}$ of $1 \times$ SSC/0.1\% SDS buffer, and then three times with $500 \mu \mathrm{l}$ of $0.1 \times \mathrm{SSC} / 0.1 \%$ SDS buffer preheated at $65^{\circ} \mathrm{C}$. The captured DNA fragments were eluted with $50 \mu \mathrm{l}$ of $0.1 \mathrm{M}$ $\mathrm{NaOH}$ and transferred to a sterile tube containing $70 \mu \mathrm{l}$ of $1 \mathrm{M}$ Tris$\mathrm{HCl}$ pH 7.5 buffer. Captured libraries were finally PCR-amplified with 25 cycles using primers fully complementary to Illumina 


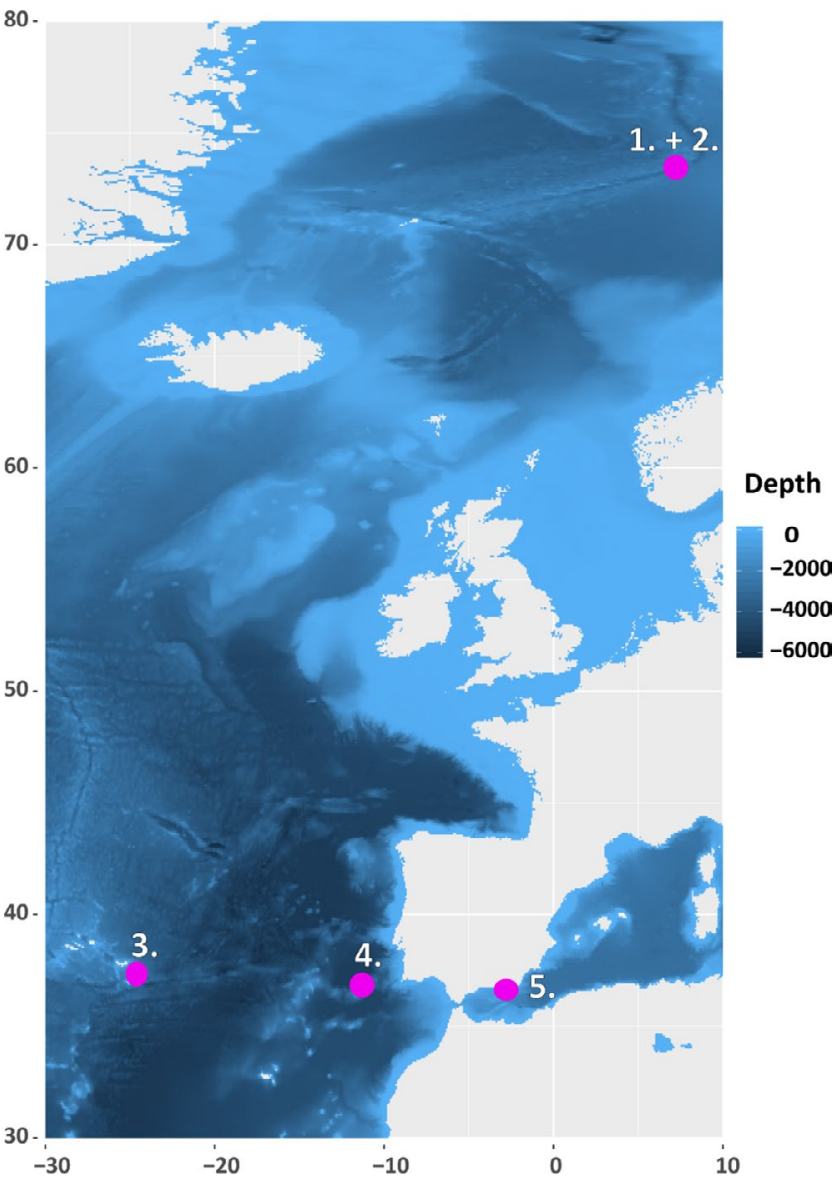

FIGURE 1 Map showing the five stations used for sampling in this study, with hydrothermal vents at stations 1 and 2 as well as seamounts at the others. The information for the stations and samples is presented in Table 1

adapters. To increase the enrichment efficiency, the second round of capture was performed from the first-round capture products. Captured libraries were then sequenced with two Illumina MiSeq $2 \times 300$ bp runs.

\section{3 | PCR-based metabarcoding}

Metabarcoding was performed at the National Center of Sequencing (Genoscope, Paris, France) following protocols published by Brandt et al. (2021). PCR was performed on two barcoding gene regions (Table 3) targeting prokaryotes (V4 16S rDNA region) or eukaryotes (V1-V2 18S rDNA region). Each $25 \mu \mathrm{l}$ PCR contained Phusion HighFidelity PCR $2 \times$ Master Mix (GC buffer for $18 \mathrm{~S}$ and HF buffer for 16SV4; New England Biolabs), each primer at $0.5 \mu \mathrm{M}$, and approximately 10-20 ng of DNA template and was brought to volume with molecular water. The PCR cycling conditions were $98^{\circ} \mathrm{C}$ for $30 \mathrm{~s}$, followed by 30 (for $18 \mathrm{~S}$ ) or 29 (for $16 \mathrm{SV} 4$ ) cycles of $98^{\circ} \mathrm{C}$ for $10 \mathrm{~s}$, annealing at $50^{\circ} \mathrm{C}$ for $45 \mathrm{~s}, 72^{\circ} \mathrm{C}$ for $60 \mathrm{~s}$, and final elongation at $72^{\circ} \mathrm{C}$ for 10 min. Each library was prepared using triplicate PCR with a Kapa Hifi HotStart NGS library amplification kit (Kapa Biosystems), and triplicates were pooled for sequencing with HiSeq technology in $2 \times 250$ bp reading mode.

\section{4 | Sequencing data processing and analysis}

\subsection{1 | Capture}

Raw reads were trimmed for Illumina adapters using Trimmomatic (v 0.38; Bolger et al., 2014) and then quality-filtered with PRINSEQ-lite PERL script (min_qual_mean $=25$, trim_qual_window $=3$, trim_qual_ step $=1$, min_len $=60 ;$ Schmieder $\&$ Edwards, 2011). Trimmed reads corresponding to rDNA were extracted using SortMeRNA (v2.1; Kopylova et al., 2012) with default parameters. Near-full-length $16 \mathrm{~S}$ and $18 \mathrm{~S}$ rDNA sequences were reconstructed using EMIRGE software (v 0.60; Miller et al., 2011) and the emirge_amplicon.py script. This tool allows reference-based assembly of reads while allowing the reconstruction of distant variants and avoiding the generation of chimeras, despite reports of chimeric sequences in reference databases that may lead to assignment issues and a call for "strict database prescreening" that "should eliminate this potential problem". The database used was SILVA 132 SSURef NR99, including fragments with lengths from 1200-2000 bp. The parameters used were join_threshold fixed to 1 and 120 iterations. Only sequences longer than 800 bp were kept. Taxonomic affiliation was performed using the plugin "feature-classifier sklearn classifier" from QIIME2 (v. 2019.1; Bokulich et al., 2018; Bolyen et al., 2019) and the fulllength SILVA 132 database, with the $p$-confidence set to 0.7. This type of analysis is further referred to as $\mathrm{CBH}$-long.

Additionally, Kraken2-based analysis (Wood et al., 2019; Wood \& Salzberg, 2014) was performed starting from paired reads to evaluate all captured diversity (without gene reconstruction), as too low coverage of some taxa could hinder the possibility of reconstructing longer sequences and thus cause the lack of these taxa in the final data set. The database used was the prepackaged SILVA database provided by Kraken2. We tested the confidential score from 0.0 to 1.0 with 0.1 steps. For the final analyses, a score of 0.7 was retained, ensuring good specificity of taxonomic affiliation. This is in line with a previous report that values from 0.6 up to 0.7 indicated the best results for sensitivity and precision (Wood \& Salzberg, 2014). Data related to these analyses are further mentioned as $\mathrm{CBH}$-short.

\subsection{2 | PCR-based metabarcoding}

The bioinformatic pipeline applied is described in Brandt et al. (2021). Primers and leftover adapters were removed with the program Cudadapt (Martin, 2011). The basic pipeline was then based on DADA2 using stringed error correction (Callahan et al., 2016), including fragment size selection with an expected length of 300500 bp for both 16SV4 and 18SV1V2, followed by a chimera removal step. Sequences were then clustered into operational taxonomic units (OTUs) using the program swarm2 (Mahé et al., 2015). As in the 
TABLE 2 Probes used in this study; the $16 \mathrm{~S}$ probe was designed by Gasc and Peyret (2018), and the $18 \mathrm{~S}$ probe was designed in this study

\begin{tabular}{|c|c|}
\hline Name & Sequence $3^{\prime}-5^{\prime}$ \\
\hline 16S_1 & CCAGACTCCTACGGGAGGCAGCAGTGGGGAA \\
\hline $16 S_{-} 2$ & AAACTCCTACGGGAGGCAGCAGTGGGGAATCT \\
\hline 16S_3 & CRAACSGGATTAGATACCCSGGTAGTCC \\
\hline $16 S_{-} 4$ & AACAGGATTAGATACCCTGGTAGTCCACGCC \\
\hline $16 S_{-} 5$ & GGGAGCAAACAGGATTAGATACCCTGGTAGT \\
\hline $16 S_{-} 6$ & AACAGGATTAGATACCYTGGYAGTCCACGC \\
\hline $16 S_{-} 7$ & AACAGGATWAGATACCCKGGYAGTCCAYRC \\
\hline $16 S \_8$ & ACTCAAAGGAATTGACGGGGGCCCGCACAAG \\
\hline 16S_9 & CACAAGCGGTGGAGCATGTGGTTTAATTCGA \\
\hline $16 S_{-} 10$ & CGCAAGDRTGAAACTTAAAGGAATTGGCGGGGGAGCAC \\
\hline $16 S \_11$ & GTTGGGTTAAGTCCCGCAACGAGCGCAACCC \\
\hline $16 S \_12$ & GAGAGGWGGTGCATGGCCGYCGYCAGYTCGT \\
\hline $16 S \_13$ & CATGGTTGTCGTCAGCTCGTGTCGTGAGATG \\
\hline $16 S \_14$ & TGTCGTCAGCTCGTGTCGTGAGATGTTGGGTTAAGTCCCGCAACGAGCSS \\
\hline $16 S \_15$ & TCGTCAGCTCGTGTYGTGAGRTGTTSGGTTAAGTCC \\
\hline 18S_1 & AGGGCAAGTCTGGTGCCAGCAGCCGCGGTAA \\
\hline $18 S_{-} 2$ & TCTGGTGCCAGCAGCCGCGGTAATTCCAGCT \\
\hline 18S_3 & TGCCAGCAGCCGCGGTAATTCCAGCTCCAAT \\
\hline $18 S_{-} 4$ & CGCGGTAATTCCAGCTCCAATAGCGTATATT \\
\hline $18 S \_5$ & GAGGGCAAGTCTGGTGCCAGCAGCCGCGGTAATTCCAGCTCCAATAGCGT \\
\hline $18 S_{-} 6$ & GTCCCTGCCCTTTGTACACACCGCCCGTCGC \\
\hline 18S_7 & GATTACGTCCCTGCCCTTTGTACACACCGCC \\
\hline $18 S_{-} 8$ & TTGATTACGTCCCTGCCCTTTGTACACACCGCCCGTCGCTA \\
\hline 18S_9 & GAGCCTGCGGCTTAATTTGACTCAACACGGG \\
\hline 18S_10 & AAGGAATTGACGGAAGGGCACCACCAGGAGT \\
\hline $18 S \_11$ & GGAAGGGCACCACCAGGAGTGGAGCCTCGGCTTAATTTGACTCAACACGG \\
\hline $18 S \_12$ & TGGTGGTGCATGGCCGTTCTTAGTTGGTGGA \\
\hline $18 S \_13$ & TGGGTGGTGGTGCATGGCCGTTCTTAGTTGGTGGAGTGATTTGTCT \\
\hline 18S_14 & GCAATAACAGGTCTGTGATGCCCTTAGATGT \\
\hline $18 S \_15$ & AAACTTAAAGGAATTGACGGAAGGGCACCAC \\
\hline $18 S \_16$ & GGGGGAGTATGGTCGCAAGGCTGAAACTTAA \\
\hline 18S_17 & GTATGGTCGCAAGGCTGAAACTTAAAGGAATTGACGGAAGGGCACCACCA \\
\hline
\end{tabular}

initial pipeline (Brandt et al., 2021), we used an iterative local threshold ( $d$ ) of 1 for 16SV4, whereas we increased it to $d=3$ for 18SV1V2, as the low number of samples in this experiment did not allow the rational use of the postclustering curation algorithm Lulu (Frøslev et al., 2017). The ribosomal sequences were taxonomically assigned against the Silva database (release 132; Pruesse et al., 2007) with the MegaBlast algorithm (using a query coverage per subject of 80 and the best hit chosen by identity with a minimum of 70; Camacho et al., 2009).

A decontamination step was applied after clustering, using extraction and PCR negative controls using decontam (Davis et al., 2018), by the prevalence method with a threshold of 0.8. Finally, all OTU counts were adjusted using an R-based abundance renormalization procedure (Wangensteen et al., 2018) to renormalize potential tag switches during library preparations (Schnell et al., 2015).

\section{5 | Formatting data and statistics}

Although the same reference databases were used, the taxonomic lineage descriptions are not harmonized for all entries, and descriptions such as phylum and class can differ between entries for the same taxon. Therefore, each individual identified was assigned to its taxonomic ID lineage based on the NCBI taxonomy database (Sayers et al., 2020) to compare the same taxonomic levels among and within methods.

Statistical test were performed with $R$ ( $R$ version 3.4.4). To compare relative abundance data, the count of sequences was standardized to the total number of reads per sample (decostand function, divide by the margin total). Similarity analyses were performed through a pairwise Mantel test based on the Bray-Curtis beta-diversity metric using vegan (Oksanen et al., 2008) for both 


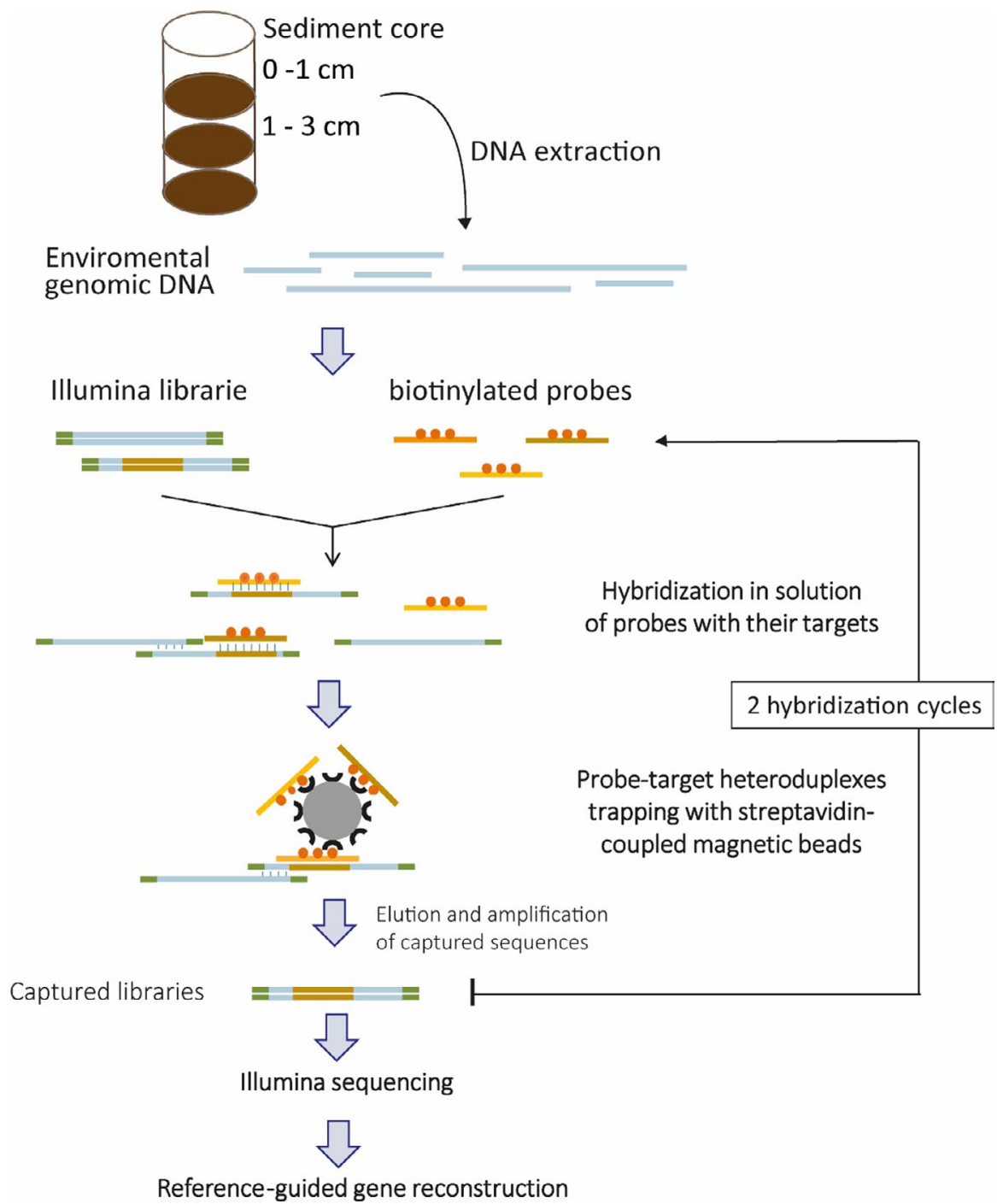

FIGURE 2 Illustration of the capture by hybridization $(\mathrm{CBH})$ approach applied in this study

TABLE 3 Primers used for PCR enrichment. The barcode region, amplicon length, sequence, and first publication reference are included

\begin{tabular}{llllll} 
Name & Direction & Region & A. size bp & Primer sequence 5'-3' & Reference \\
SSUF04 & Forward & 18S V1-V2 & 356 & GCTTGTCTCAAAGATTAAGCC & (Blaxter et al., 1998) \\
SSURmod & Reverse & 18S V1-V2 & & CCTGCTGCCTTCCTTRGA & (Sinniger et al., 2016) \\
$515 F-Y$ & Forward & $16 S$ V4 & 411 & GTGYCAGCMGCCGCGGTAA & (Parada et al., 2016) \\
$926 R$ & Reverse & 16S V4 & & CCGYCAATTYMTTTRAGTTT & (Parada et al., 2016) \\
\hline
\end{tabular}

prokaryotic (16S) and metazoan (18S) communities. The homogeneity of sample dispersion was analysed using the betadisper function (distances to centroid) and tested for significance using analysis of variance (ANOVA).

\section{3 | RESULTS}

\subsection{Probes for $\mathrm{CBH}$}

In addition to the $1516 \mathrm{~S}$ rDNA probes available, 17 explorative probes targeting $18 \mathrm{~S}$ rDNA were designed to recover a broad range of eukaryotes, with a special emphasis on metazoans. Probe positions within $16 \mathrm{~S}$ and $18 \mathrm{~S}$ rDNA genes are visualized in Appendix S2. The coverage of the two sets of probes, determined in silico, reached $99.99 \%$ for $16 \mathrm{~S}$ rDNA and $99.98 \%$ for $18 \mathrm{~S}$ rDNA of the 369,953 and 55,145 targeted taxa present in the SILVA SSU NR99 (version 132; see Appendix S3), indicating a high potential sensitivity of probes for the recovery of prokaryotic and eukaryotic diversity.

\section{2 | Sequencing output and fragment length}

$\mathrm{CBH}$ resulted in 2,231,139 to $12,799,906$ reads per sample. After the first filtering and trimming steps, approximately 7 to $34 \%$ were identified as rDNA (general sequencing output given in Appendix 
S4). For PCR-based metabarcoding (MTB), a total of 288,094 to $1,278,400$ sequences were obtained per sample, yielding 2231 to 4133 OTUs for 16 SV4 and 770 to 2169 for 18SV1V2 per sample. The $\mathrm{CBH}$ data were analysed by two different pipelines, first direct use of the short fragments (hereafter referred to as $\mathrm{CBH}$-short) with Kraken 2, using the unaligned reads with a mean length of 200 to $289 \mathrm{bp}$, which was shorter than the fragments of up to $450 \mathrm{bp}$ obtained with PCR-based metabarcoding. Second, EMIRGE was used to reconstruct "full barcodes" (hereafter named CBH-long) allowed the reconstruction of fragments of on average 731 near-full length markers per sample, reaching up to 1200 to 1450 bp for archaea, up to $1600 \mathrm{bp}$ for bacteria and 1200 to $1900 \mathrm{bp}$ for eukaryotes (Figure 3).

\section{3 | Comparing general diversity}

As expected, the taxonomic assignment led to different outcomes depending on the analyses, as PCR-based metabarcoding results were presented as OTUs, $\mathrm{CBH}$-long results as reconstructed sequences, and $\mathrm{CHB}$-short results as grouped (not clustered) sequences for the same taxa. Interestingly, the detection trends of taxa and phyla among the methods varied across the three analysed kingdoms, Archaea, Bacteria and Eukaryota (synoptic visualization in Figure 4, samplewise data listed in Appendix S5).

In the case of Archaea CHB-long, a slightly higher number of taxa was revealed than with $\mathrm{CBH}$-short or MTB, but with one missing phylum (DPANN group). Most of this difference came from the Tack group, with 419 taxa identified by $\mathrm{CBH}$-short, 869 taxa identified using long sequences and only 166 taxa identified with MTB. For bacteria, $\mathrm{CBH}$ shows generally higher diversity detection; $\mathrm{CBH}$ short detected most phyla, with 18 and 11 using $\mathrm{CBH}$-long and 12 with MTB. Some rare phyla were only detected by $\mathrm{CBH}$-long or MTB, but all were detected by $\mathrm{CBH}$-short. Additionally, over 1200 more taxa were revealed via full-length barcodes than with OTUs by MTB. More importantly was the difference in the displayed diversity itself; over $50 \%$ of bacteria identified with $\mathrm{CBH}$ belonged to Proteobacteria (3588 taxa with $\mathrm{CBH}$-long; 10,520 taxa with
$\mathrm{CBH}$-short), and only 25\% (1228 taxa) belonged to Proteobacteria with MTB.

In contrast, using $\mathrm{CBH}$ (short and long) for eukaryotes showed opposite trends. Here, most detections of phyla and other taxa were obtained by MTB. Overall, less than $2 \%$ of the reconstructed fragments ( $n=267$ ) were identified compared to those with MTB (OTU $n=13,794$ ), and only six out of 18 phyla were identified, whereas $\mathrm{CBH}$-short still detected 14 phyla $(n=4571)$. However, these newly designed $18 \mathrm{~S}$ probes mostly focused on metazoans, as part of Opisthokonta; here, $\mathrm{CBH}$ showed a relatively higher percentage of detections. With a total of 2527 taxa identified using MTB, 2089 were $\mathrm{CBH}$-short, and 181 were full-length sequences. Finally, the overall identification and displayed biodiversity varied in the amount and abundance of phyla, depending on the kingdom/gene region as well as the analytical method.

\section{4 $\mathrm{CBH}$ for metazoans}

The identification of taxa regarding their phyla in the NCBI taxonomy is presented in Figure 5. As expected, generally higher diversity was detected by $\mathrm{CBH}$-short (Kraken2), with 21 phyla, compared to 16 with MTB. Some groups, Acanthocephala, Cycliophora, Entoprocta, Gnathostomulida, Micrognathozoa, and Nematomorpha, were only detected with $\mathrm{CBH}$, while only one group was specifically detected by PCR-based metabarcoding (Ctenophora). The greatest difference was in the number of detected nematodes, with 1074 (MTB) to 82 (CBH-short) taxa. Analysing sample-specific results showed in more detail the differences but also similarities of the different methods (Figures 6 and 7). As an example, $\mathrm{CBH}$-short showed an overwhelming number of reads for detected sponges and in sample 1A, with 20 taxa identified within all four lineages of Porifera, Calcarea, Demospongiae, Hexactinellida, and Homoscleromorpha. A total of 638,602 (over $97 \%$ of all metazoan sequences in this sample) sequences belonging to Demospongiae were detected, representing 16 different families. Within this sample, 29 different sequences of Demospongiae were reconstructed ( $\mathrm{CBH}$-long). Additionally, for the MTB, over $87 \%$ of the metazoan sequences identified in this
FIGURE 3 Query length of identified sequences with the $\mathrm{CBH}$ analysed using the EMIRGE pipeline ( $\mathrm{CBH}$-long). Metabarcoding of the same genes ranked between 313-411 base pairs (bp)

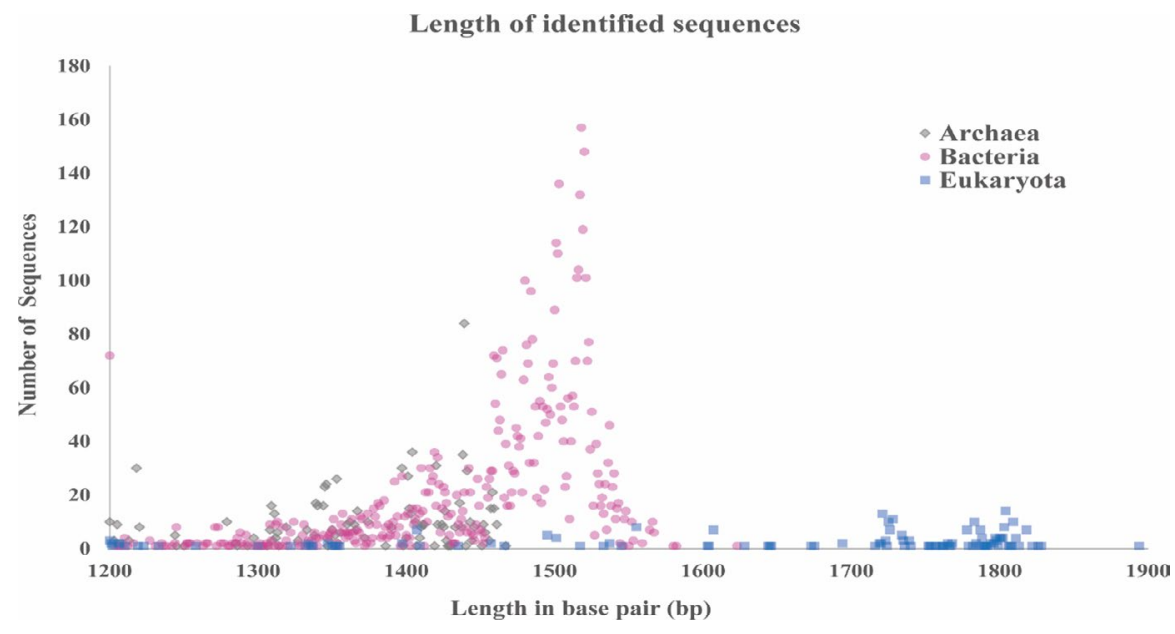



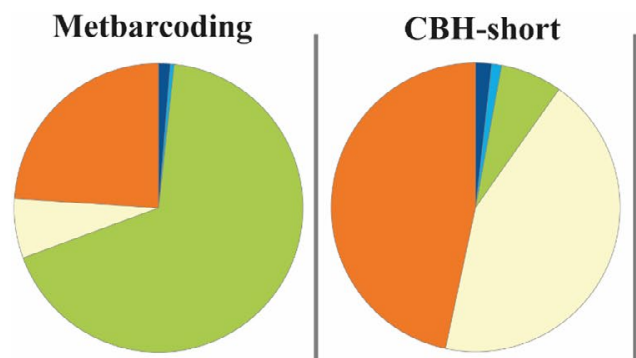

$\mathrm{N}=692$

$\mathrm{P}=5$

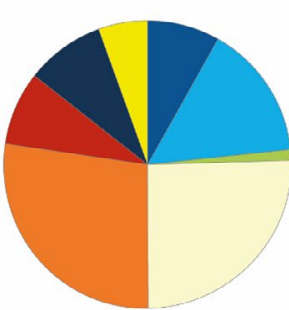

$\mathrm{N}=4880$

$\mathrm{P}=12$

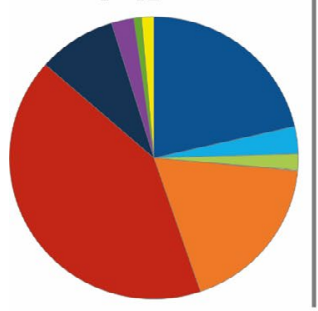

$\mathrm{N}=13794$

$\mathrm{P}=18$

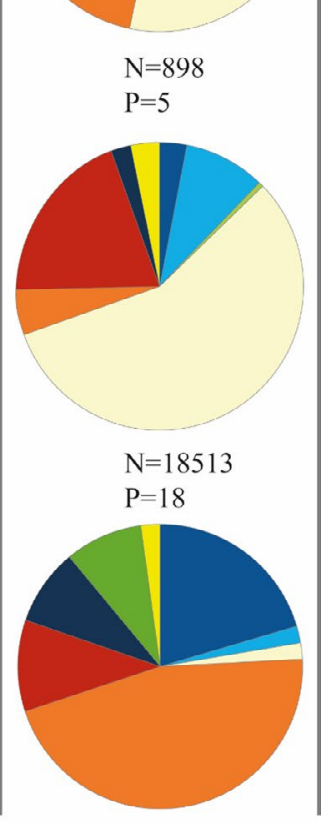

$\mathrm{N}=4571$

$\mathrm{P}=14$

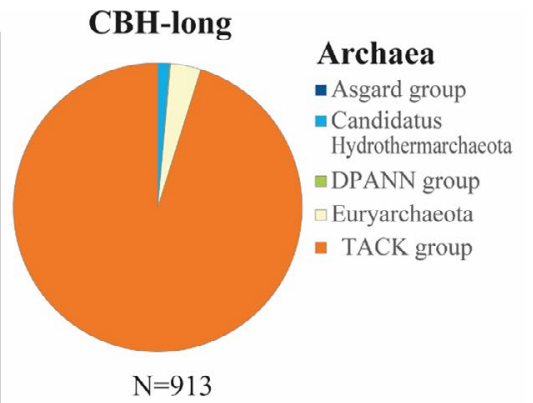

$\mathrm{P}=4$

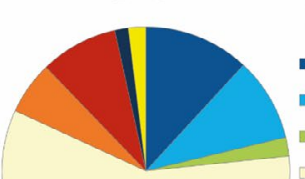

Bacteria

- Acidobacteria

$-\mathrm{FCB}$ group

Nitrospiraep

$\neg$ Proteobacteria

- $\mathrm{PVC}$ group

- Terrabacteria group

- unclassified

= Others

$\mathrm{N}=6132$

$\mathrm{P}=11$

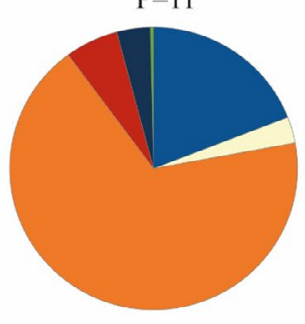

$\mathrm{N}=267$

$\mathrm{P}=6$

\section{Eukaryotes}

- Alveolata

Amocbozoa

$\square$ Apusozoa

Euglenozoa

- Opisthokonta

- Rhizaria

- Stramenopiles

- unclassified

- Viridiplantae

$\square$ Others

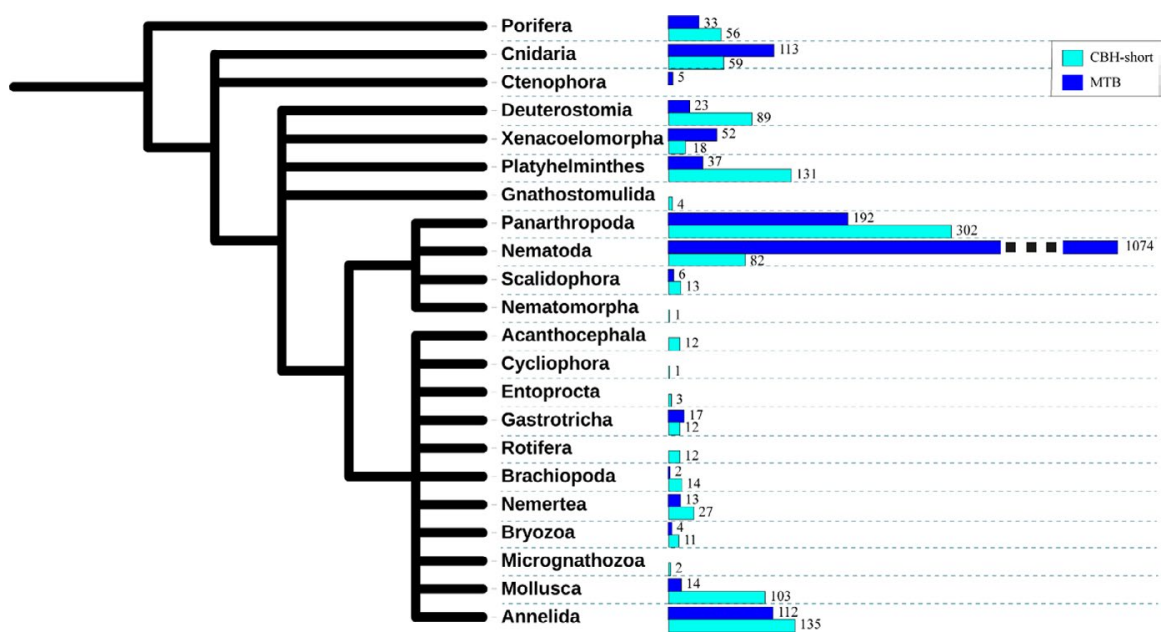

FIGURE 4 Comparison of highlevel taxonomy of all 10 samples using metabarcoding versus $\mathrm{CBH}$ with two distinct pipelines: $\mathrm{CBH}$-long (EMIRGE) and $\mathrm{CBH}$-short (Kraken2). " $\mathrm{N}$ " indicates the number of identified taxa, and "P" the number of phyla, while phyla with less than $2 \%$ occurrence were counted together as "Others"
FIGURE 5 Phylogenetic tree based on NCBI taxonomy, created by iTol. Indicated are the counted taxa per phylum, while metabarcoding is indicated with dark blue and $\mathrm{CBH}$-short with light blue particular sample were Porifera, but only one large cluster (OTU) of Demospongiae and two small calcares were detected. At the species level, 12 demospongies were identified with $\mathrm{CBH}$-long, where one overlap with the MTB approach detected species at the genus level.

\subsection{Statistical analyses comparing CBH with MTB}

The ten samples chosen for this comparative study were collected at five different sites, including two different ecosystems, seamounts (in the Atlantic Ocean and the Mediterranean) and hydrothermal vents (inactive site, characteristic of deep bathyal sediment systems), to test different biological communities. Based on multidimensional analysis of Bray-Curtis (based on the number of sequences) distances, both gene regions showed significantly heterogeneous dispersions among methods (betadisper, tested with ANOVA and methods as grouping factors, for $16 \mathrm{~S}$, sum of squares $=0.0078647$, $F$-value $=4.4288$, and $p$-value $=.02171 ;$ for $18 \mathrm{~S}$, sum of squares $=0.38879, F$-value $=$ $6.1449, p$-value $=.006319$ ). This reflects the different levels of heterogeneity in communities revealed by each method. 
Reads
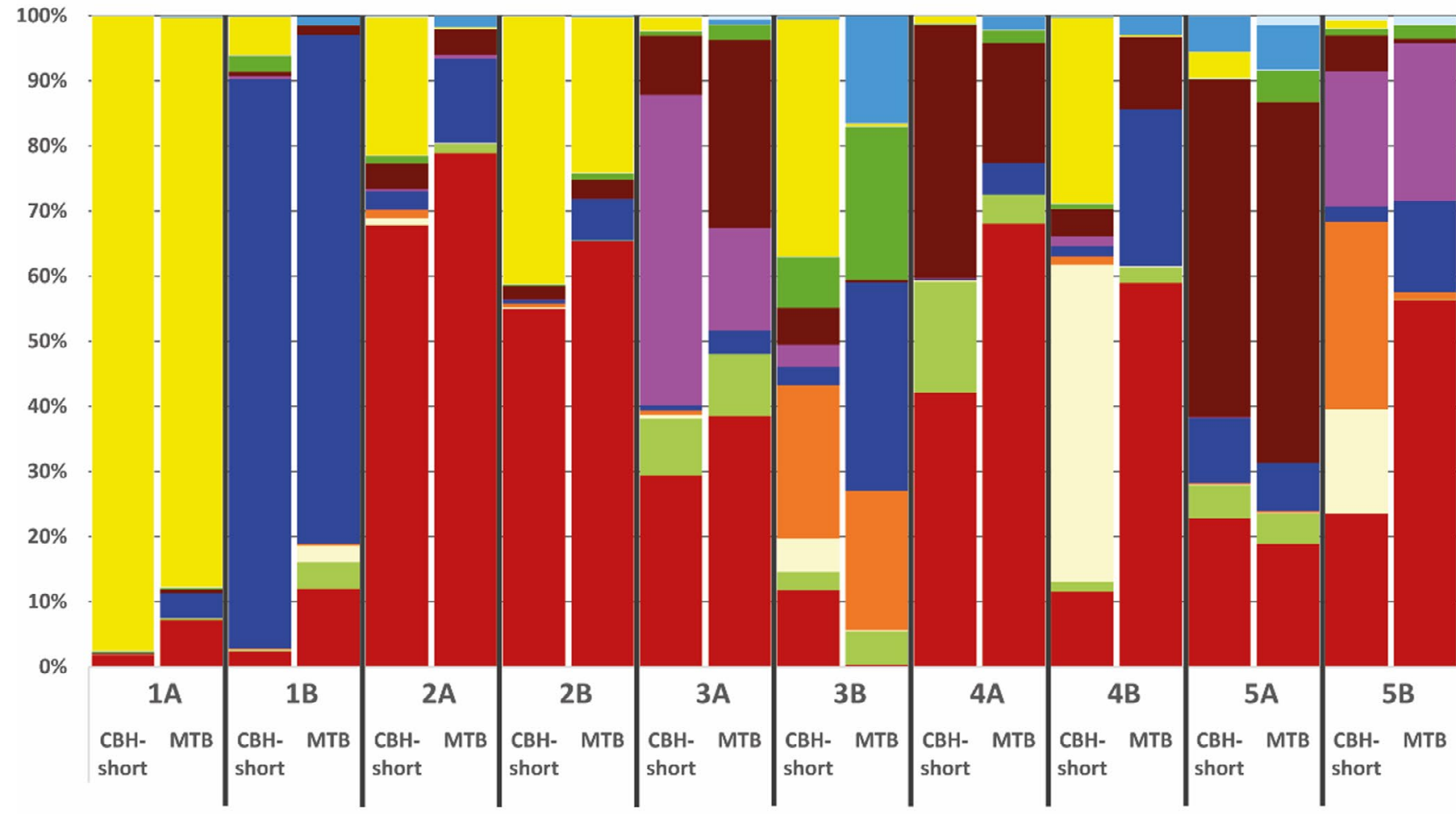

Taxa
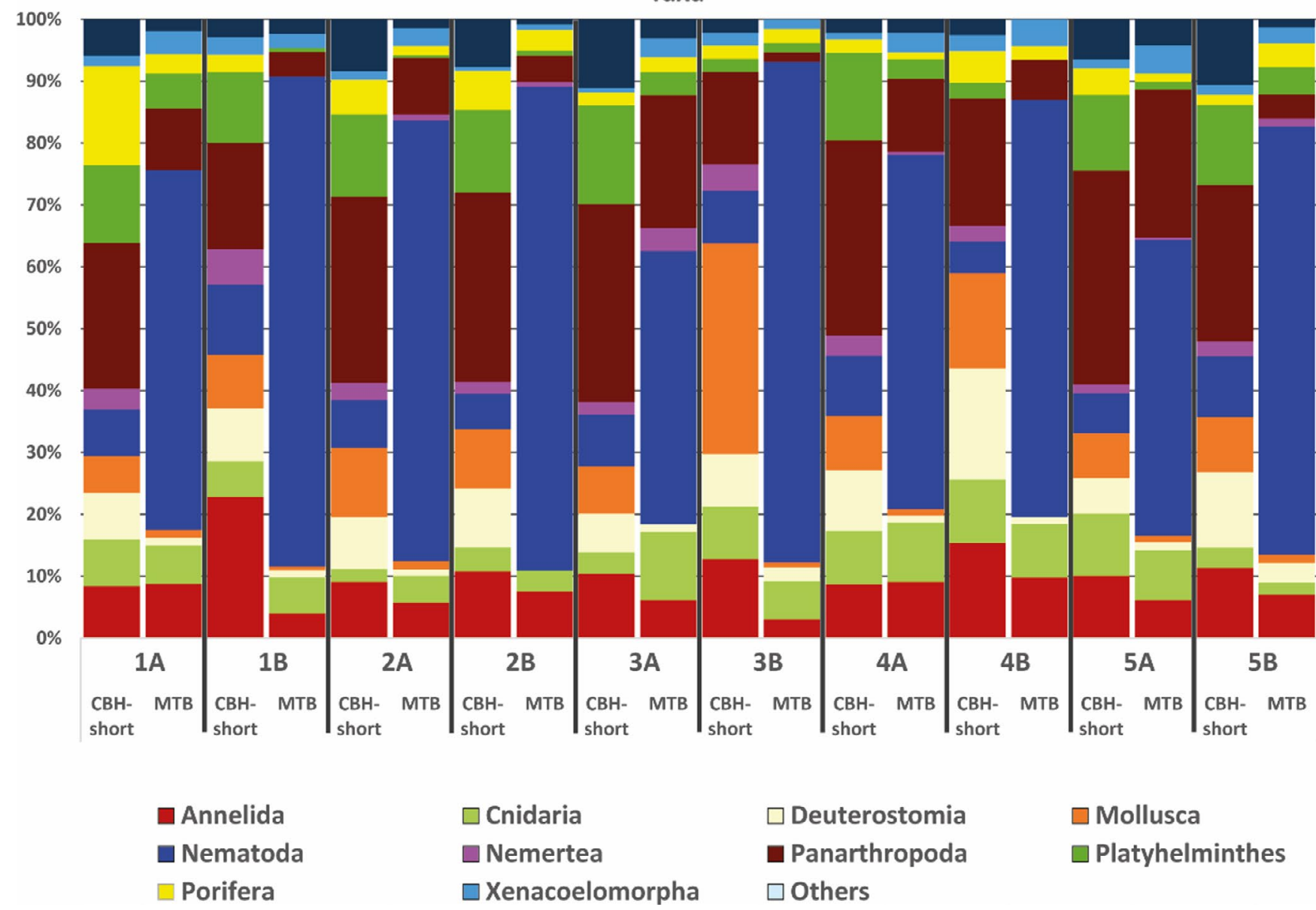
$\square$ Deuterostomia
- Panarthropoda $\square$ Others

$\square$ Mollusca

$\square$ Platyhelminthes

FIGURE 6 Samplewise detection with 18S analyses from CBH-short and metabarcoding for all detected metazoan phyla. The upper graph shows the percentage of detected taxa per phylum, and the lower shows the percentage of reads per phylum 


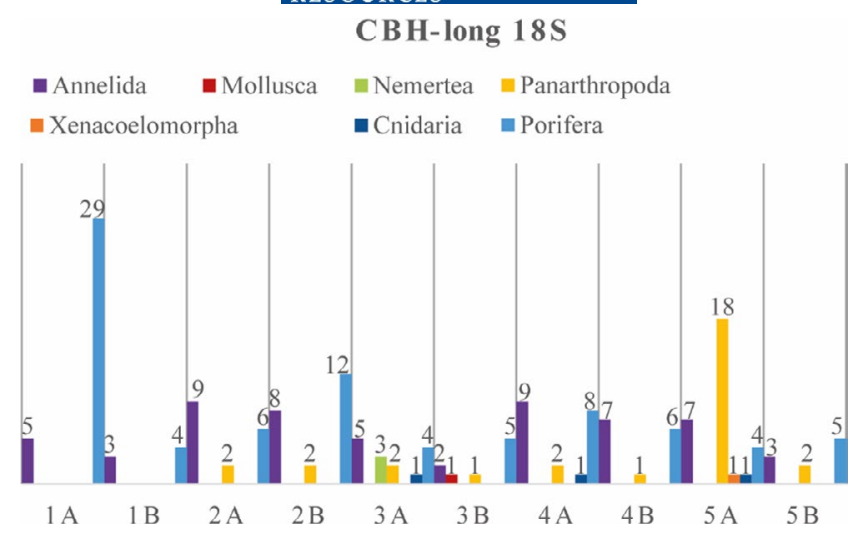

FIGURE 7 Numbers of taxa per sample using $\mathrm{CBH}$-long

For prokaryotes, the Mantel test showed that the results of all three analyses were significantly correlated (Table 4), yet all samples characterized with MTB clustered separately from those characterized with $\mathrm{CBH}$-short and $\mathrm{CBH}$-long (Figure 8a), suggesting that the methods were the dominant driver of differentiation. For metazoan detections based on 18SV1-V2, a significant correlation emerged between MTB and CBH short, and the relationship between MTB and $\mathrm{CBH}$ long was nearly significant, while no correlation was established with the Mantel test between the two $\mathrm{CBH}$ methods. The results seem to show a grouping of $\mathrm{CBH}$ long on the second axis (with the first axis illustrating well the dispersion among samples for this method), while the opposite was true for MTB (Figure 8b).

\section{4 | DISCUSSION}

Deep-sea biodiversity assessments are one of the major gaps in marine ecology and evolution and are strongly needed for international policy and management (Costa et al., 2020). Metagenomics (MTG) allows high-resolution inventories relying on strong phylogenetic reconstructions but is still too expensive and time consuming, as well as biased toward prokaryotes that represent most biomass in sediment samples (Danovaro et al., 2014). Although PCR-based metabarcoding (MTB) is relatively fast and less expensive, it suffers biases and limited resolution to the species level due to gaps in reference databases and highly conserved regions with low variability (von Ammon et al., 2018; Machida \& Knowlton, 2012; Wangensteen et al., 2018).

In this study, we show that capture by hybridization (CBH) can offer a good balance between MTG and MTB, allowing the detection of a broader spectrum of phyla than MTB, and the reconstruction of full-length barcodes of up to $2000 \mathrm{bp}$ allows the potential for robust phylogenetic reconstruction to improve the resolution of taxonomic assignments of poorly studied taxa (Wilson et al., 2018).

The approach applied here, initially developed for prokaryotes by Gasc and Peyret (2018), confirms that CBH can be adapted to describe bacterial communities in the marine realm. Furthermore, the first assay of transfer of this methodology to deep-sea metazoan communities proved successful, with five more phyla (upon 16) detected with $\mathrm{CBH}$ than MTB. This is in line with other recent $\mathrm{CBH}$ studies targeting more restrictive sets of metazoan phyla from bulk DNA, their conservative ethanol or eDNA samples (here bulk, freshwater and sediment; Gauthier et al., 2020; Giebner et al., 2020; Shokralla et al., 2016; Wilcox et al., 2018). All of these studies also demonstrated the ability of $\mathrm{CBH}$ to overcome biases due to $\mathrm{PCR}$ steps in MTB and enhance detection rates, leading to a more reliable representation of not only the species present in the samples but sometimes also of their relative biomasses, highlighting possibilities for quantitative analyses. Here, $\mathrm{CBH}$ results confirmed the improvement of biodiversity inventories allowed by direct taxonomic identification using Kraken2, which is based on exact k-mer matches and clustering of detected taxa to the lowest common ancestor (Wood $\&$ Salzberg, 2014). The sequence reads included different fragments within the $18 \mathrm{~S}$ gene and were not merged. One must thus keep in mind this may contribute to a larger amount of taxa detected with $\mathrm{CBH}$ than with MTB, where sequences are merged pairwise, filtered, clustered and are all from identical positions, yet this clearly does not explain the broadest spectra of diversity with five (>30\%) more phyla detected with $\mathrm{CBH}$ than MTB.

The second aim of this study was the reconstruction of full barcodes to detect a broader spectrum of diversity and to enable robust phylogenetic reconstruction with long sequences. This has been proven feasible. An example of successful reconstruction of long fragments and increased resolution is the case of Porifera taxa in sample 1A. While MTB detected only 3 taxa, 20 identifications assigned to distinct taxa were recorded with $\mathrm{CBH}$-short, resulting in 29 long sequences with $\mathrm{CBH}$-long, assigned to 12 clearly identified species. The different hypervariable regions of $18 \mathrm{~S}$ used for MTB vary in their resolution for the different phyla and nucleotide positions (Machida \& Knowlton, 2012); using a full barcode thus clearly improves the number and quality of detected taxa, as exemplified in sample 1A for Porifera. The full $18 \mathrm{~S}$ should thus be targeted to obtain the best out of the two objectives of $\mathrm{CBH}$ : (i) increase the breadth of phyla detected and improve their taxonomic identification, and (ii) enable several robust phylogenetic and phylogeographic approaches to describe and unravel the biodiversity of deep-sea communities. In addition, focusing on longer fragments will limit sources of "contamination" and focus on contemporary communities by limiting the contribution of extracellular DNA from marine sediments (Torti et al., 2015).

\section{1 | Perspectives on improvement}

Taking full advantage of the potential of $\mathrm{CBH}$ will require several important improvements to focus on biodiversity inventories based on long and resolutive fragments: adapting sequencing depth and testing of de novo DNA reconstruction.

In fact, the reconstruction was done here by mapping sequences to the existing SILVA databases using the program EMIRGE (Miller et al., 2011). This program developed for $16 S$ has been shown to be 
extremely efficient, with 2224 full barcodes reconstructed, leading to 6132 detections in 10 samples. Theoretically, a similar result could be expected for eukaryotic 18S, yet the numbers of identifications with EMIRGE were less than $6 \%$ compared to those with short $\mathrm{CBH}$ with Kraken2. To exclude the possibility that EMIRGE is not sufficient for $18 \mathrm{~S}$ reconstruction, we also tested the program MAFFT for reconstruction (Katoh et al., 2002), which was also shown to be suitable, with similar results (data not presented). As sequencing depth has an effect on the coverage of targeted sequences and influences gene reconstruction (Pericard et al., 2018), we suggest that a much greater sequencing depth than that used in this first assay will be required to improve the reconstruction of long fragments based on $\mathrm{CBH}$. For eDNA analysis in general, high sequencing depth is crucial (Singer et al., 2019) and will allow de novo assembly instead of mapping algorithms, improving species identification (Deiner et al., 2017) and the detection of taxa or groups absent from the reference data set. In this first study, both gene regions were pooled for sequencing, and the results suggest (i) that the $16 \mathrm{~S}$ probes were more efficient than the newly designed $18 \mathrm{~S}$ probes in uncovering community richness, (ii) the dominance of prokaryotic biomass was reflected, or (iii) the method suffered from both limitations. The unbalanced biomass could be fixed by sequencing libraries from each rDNA separately. However, most bacterial diversity may be revealed with lower sequencing depth for these unicellular organisms, while the higher variations in body size and of the number of rDNA $18 \mathrm{~S}$ copies among metazoans may result in a more uneven distribution of the number of fragments among taxa. In any case, a higher sequencing depth will be needed to unravel the diversity of the communities they form through full barcode reconstruction. Setting a generic number

TAB LE 4 Results of the pairwise Mantel tests based on the Bray-Curtis metric

\begin{tabular}{|c|c|c|c|c|}
\hline & \multicolumn{2}{|l|}{$16 S$} & \multicolumn{2}{|l|}{$18 S$} \\
\hline & $r$ & $p$-Value & $r$ & $p$-Value \\
\hline CHB-short: $\mathrm{CBH}$-long & 0.7262 & 0.006 & 0.2188 & 0.172 \\
\hline CHB-short: MTB & 0.7601 & 0.001 & 0.4727 & 0.019 \\
\hline CBH-long: MTB & 0.5814 & 0.005 & 0.4579 & 0.0565 \\
\hline
\end{tabular}

Note: Numbers in bold reflect significant $p$-values. of sequences would not be realistic because the optimal number strongly depends on the diversity and biomass of the standing stock in the sediment. When studying new areas, pilot metabarcode studies may help tune the sequencing depth, allowing the optimal inventory of full-length metabarcodes in the studied ecosystems.

We tested here for the first time a new set of DNA probes that proved useful and efficient, yet the method still needs improvement to capture several taxa, such as nematodes and some other meiofauna taxa with low levels of detection. In fact, meiofauna of deepsea sediments are generally dominated by nematodes and copepods in terms of biomass, abundance, and species richness (Zeppilli et al., 2018). Neither CBH nor MTB consistently reflects this, suggesting that not only sequencing depth but also the versatility of the set of probes needs to be enhanced.

Finally, the huge gaps in knowledge of marine biodiversity, magnified in the deep sea, result in a paucity of deep-sea sequences in nucleotide reference databases (Sinniger et al., 2016), inhibiting the efficiency of reference-based bioinformatic reconstructions (Mendoza et al., 2014). This probably explains the very uneven reconstruction success across the phyla observed, with rather good results for Porifera, Annelida, and some Arthropods, while for other phyla such as Deuterostomia, Mollusca, Nematoda, Cnidaria, and Platyhelminthes, no reconstruction could be obtained despite numerous identified sequences.

\section{2 | Cost factor}

One of the reasons for developing PCR-based metabarcoding as a future biomonitoring tool is the decreased costs (both in terms of time and money) compared to full metagenome reconstruction through shotgun sequencing or classical morphological approaches. Based on recent calculations for marine biomonitoring, the MTB approach would represent only approximately half of the costs and less than $70 \%$ of the time compared to morphological assessments (Aylagas et al.,2018). A similar study showed a significant increase in metazoan biodiversity detection with $\mathrm{CBH}$ despite it being eight times more expensive than the MTB approach (Giebner et al., 2020), which we believe may evolve as it becomes more commonly used and its protocols are optimized. We found that for $\mathrm{CHB}$, the laboratory time (a)

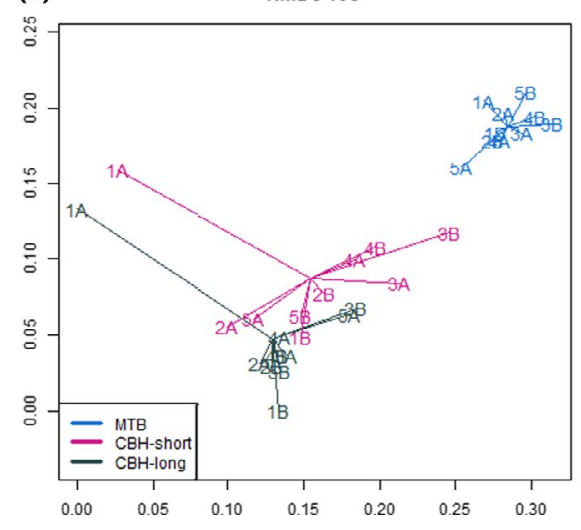

(b)

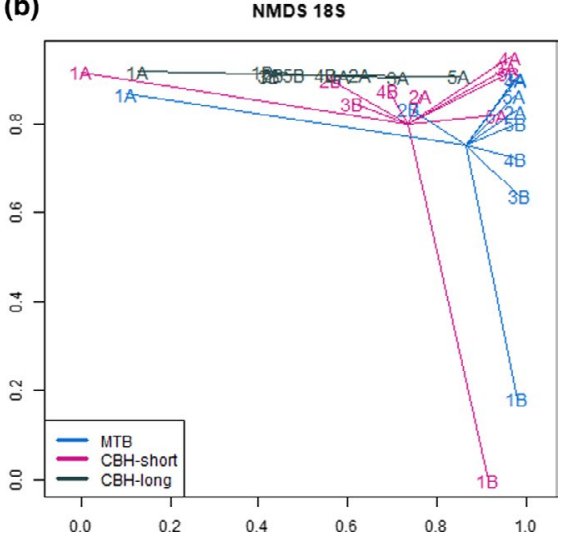

FIGURE 8 Multidimensional analysis of $16 \mathrm{~S}(\mathrm{a})$ and $18 \mathrm{~S}(\mathrm{~b})$ dissimilarity metrics obtained with $\mathrm{CBH}$ using $\mathrm{CBH}$-long, $\mathrm{CBH}$ short and MTB, based on Bray-Curtis distances 
effort is nearly the same, but the costs are at present still higher than those of MTB, although considerably lower than those of metagenome reconstruction. The major factor is the preparation of sequencing libraries per sample for $\mathrm{CBH}$ compared to MTB. In addition, although multiplexing library protocols help increase cost efficiency (for example, Förster et al., 2018; Meyer \& Kircher, 2010), the results presented here show that full metabarcodes must be targeted to obtain satisfying read lengths and taxonomic assignments, and multiplexing cannot encompass as many libraries in a sequencing lane as MTB, with over 100 samples. The computational power of these methods is also comparable: reconstruction can be demanding, yet error correction for high-quality PCR-based metabarcoding is also time- and resource-consuming. Nevertheless, as in other recent comparable studies (Liu et al., 2016; Seeber et al., 2019), we see the advantages of overcoming the slightly higher prices in the long term. In particular, using $\mathrm{CBH}$ for baseline studies can improve local reference databases thanks to improved taxonomic inferences through phylogenetic reconstruction, which will be particularly important for less described areas and taxa. These extended libraries will in turn contribute to improving the output of massive PCR-based metabarcoding screenings.

\section{5 | CONCLUSIONS}

This study presents the potential advantages of using capture-based target gene enrichment for biodiversity assessment of prokaryotic and eukaryotic communities, with a specific application in poorly known deep-sea benthic ecosystems. The results showed that capture-based target gene enrichment has the potential for considerable added value compared to PCR-based metabarcoding in performing referenced biodiversity inventories and phylogenetic reconstruction, improving knowledge for both biomonitoring and management purposes. $\mathrm{CBH}$ showed the ability to reach the two main goals initially established in this study: (i) revealing a broader spectrum of metazoan diversity and (ii) reconstructing full-length barcode regions (up to $1900 \mathrm{bp}$ ) allowing better phylogenetic reconstruction, thus improving taxonomic assignments. Consequently, provided sequencing depth is sufficient to allow de novo reconstruction, $\mathrm{CBH}$ can be applied at slightly higher costs than MTB for (i) identifying taxa that are not well represented (nor any close relatives) in databases, and (ii) performing phylogenetic and phylogeographic studies.

\section{ACKNOWLEDGEMENTS}

We are grateful to Projects "Pourquoi Pas les Abysses (we acknowledge Sébastien Ybert and Chantal Cahu for help in managing the project), Atlas, and Total for funding. We would also like to acknowledge the mission chiefs, crews and teams for sampling conducted during the cruises Medwaves and MarMine (MarMine; Eva Ramirez Llodra, NRC- BI Project; no. 247626/O30). We thank Miriam Brandt and Diane Bailleul for help in the early steps of this study, as well as one anonymous referee and Tristan Cordier for useful comments on a previous version of this manuscript. Founded by Total SE, Contract no. 9194 TOTAL FR0000. The MEDWAVES cruise was organized in the framework of the ATLAS Project, supported by the European Union 2020 Research and Innovation Programme under grant agreement number 678760 .

\section{AUTHOR CONTRIBUTIONS}

Sophie Arnaud-Haond and Pierre Peyret designed and directed the project. Thomas Merzi and Philippe Blanc funded and helped supervise the project. Clémence Defois, Sophie Marre and Babett Günther performed the experiments and primary data analyses. All authors provided critical feedback and helped shape the research, analysis and manuscript.

\section{DATA AVAILABILITY STATEMENT}

All original fastq.gz sequencing files have been deposited in the NCBI open access sequence read archive (SRA) under accession numbers ERS3168383, ERS3168384, ERS3168414, ERS3168415, ERS3168569, ERS3168570, ERS3168460, ERS3168461, ERS3168762, ERS3168763(MTB); and SRR13482776-SRR13482785 (CBH). All metadata associated with the samples, including detailed accession numbers, are given in Appendix S1 and are available at https://www.pangaea.de/ and BioSamples.

\section{ORCID}

Babett Günther (iD https://orcid.org/0000-0003-1225-4262

\section{REFERENCES}

Acinas, S. G., Sarma-Rupavtarm, R., Klepac-Ceraj, V., \& Polz, M. F. (2005). PCR-induced sequence artifacts and bias: Insights from comparison of two 16S rRNA clone libraries constructed from the same sample. Applied and Environmental Microbiology, 71(12), 8966-8969. https:// doi.org/10.1128/aem.71.12.8966-8969.2005

Ammon, U. V., Wood, S. A., Laroche, O., Zaiko, A., Tait, L., Lavery, S., Inglis, G. J., \& Pochon, X. (2018). Combining morpho-taxonomy and metabarcoding enhances the detection of non-indigenous marine pests in biofouling communities. Scientific Reports, 8(1), 1-11. https://doi.org/10.1038/s41598-018-34541-1

Andruszkiewicz, E. A., Starks, H. A., Chavez, F. P., Sassoubre, L. M., Block, B. A., \& Boehm, A. B. (2017). Biomonitoring of marine vertebrates in Monterey Bay using eDNA metabarcoding. PLoS One, 12(4), e0176343. https://doi.org/10.1371/journal.pone.0176343

Apothéloz-Perret-Gentil, L., Cordonier, A., Straub, F., Iseli, J., Esling, P., \& Pawlowski, J. (2017). Taxonomy-free molecular diatom index for high-throughput eDNA biomonitoring. Molecular Ecology Resources, 17(6), 1231-1242. https://doi.org/10.1111/1755-0998.12668

Armbrecht, L., Hallegraeff, G., Bolch, C. J. S., Woodward, C., \& Cooper, A. (2021). Hybridisation capture allows DNA damage analysis of ancient marine eukaryotes. Scientific Reports, 11(1), 1-14. https://doi. org/10.1038/s41598-021-82578-6

Aylagas, E., Borja, Á., Muxika, I., \& Rodríguez-Ezpeleta, N. (2018). Adapting metabarcoding-based benthic biomonitoring into routine marine ecological status assessment networks. Ecological Indicators, 95, 194-202. https://doi.org/10.1016/j.ecolind.2018.07.044

Blaxter, M. L., De Ley, P., Garey, J. R., Liu, L. X., Scheldeman, P., Vierstraete, A., Vanfleteren, J. R., Mackey, L. Y., Dorris, M., Frisse, L. M., Vida, J. T., \& Thomas, W. K. (1998). A molecular evolutionary framework for the phylum Nematoda. Nature, 392(6671), 71-75. https://doi. org/10.1038/32160 
Bohan, D. A., Vacher, C., Tamaddoni-Nezhad, A., Raybould, A., Dumbrell, A. J., \& Woodward, G. (2017). Next-generation global biomonitoring: Large-scale, automated reconstruction of ecological networks. Trends in Ecology \& Evolution, 32(7), 477-487. https://doi. org/10.1016/j.tree.2017.03.001

Bohmann, K., Evans, A., Gilbert, M. T. P., Carvalho, G. R., Creer, S., Knapp, M., Yu, D. W., \& de Bruyn, M. (2014). Environmental DNA for wildlife biology and biodiversity monitoring. Trends in Ecology \& Evolution, 29(6), 358-367. https://doi.org/10.1016/j.tree.2014.04.003

Bokulich, N. A., Kaehler, B. D., Rideout, J. R., Dillon, M., Bolyen, E., Knight, R., Huttley, G. A., \& Gregory Caporaso, J. (2018). Optimizing taxonomic classification of marker-gene amplicon sequences with QIIME 2's q2-feature-classifier plugin. Microbiome, 6(1), 90. https:// doi.org/10.1186/s40168-018-0470-z

Bolger, A. M., Lohse, M., \& Usadel, B. (2014). Trimmomatic: A flexible trimmer for Illumina sequence data. Bioinformatics, 30(15), 21142120. https://doi.org/10.1093/bioinformatics/btu170

Bolyen, E., Rideout, J. R., Dillon, M. R., Bokulich, N. A., Abnet, C. C., AlGhalith, G. A., Alexander, H., Alm, E. J., Arumugam, M., Asnicar, F., Bai, Y., Bisanz, J. E., Bittinger, K., Brejnrod, A., Brislawn, C. J., Brown, C. T., Callahan, B. J., Caraballo-Rodríguez, A. M., Chase, J., ... Caporaso, J. G. (2019). Reproducible, interactive, scalable and extensible microbiome data science using QIIME 2. Nature Biotechnology, 37(8), 852-857. https://doi.org/10.1038/s41587-019-0209-9

Bragalini, C., Ribière, C., Parisot, N., Vallon, L., Prudent, E., Peyretaillade, E., Girlanda, M., Peyret, P., Marmeisse, R., \& Luis, P. (2014). Solution hybrid selection capture for the recovery of functional full-length eukaryotic cDNAs from complex environmental samples. DNA Research, 21(6), 685-694. https://doi.org/10.1093/dnares/dsu030

Brandt, M. I., Trouche, B., Quintric, L., Günther, B., Wincker, P., Poulain, J., \& Arnaud-Haond, S. (2021). Bioinformatic pipelines combining denoising and clustering tools allow for more comprehensive prokaryotic and eukaryotic metabarcoding. Molecular Ecology Resources, 21(6), 1904-1921. https://doi.org/10.1111/1755-0998.13398

Callahan, B. J., McMurdie, P. J., Rosen, M. J., Han, A. W., Johnson, A. J., \& Holmes, S. P. (2016). DADA2: High-resolution sample inference from Illumina amplicon data. Nature Methods, 13(7), 581-583. https://doi.org/10.1038/nmeth.3869

Camacho, C., Coulouris, G., Avagyan, V., Ma, N., Papadopoulos, J., Bealer, K., \& Madden, T. L. (2009). BLAST+: Architecture and applications. BMC Bioinformatics, 10, 421. https://doi. org/10.1186/1471-2105-10-421

Cordier, T., Forster, D., Dufresne, Y., Martins, C. I. M., Stoeck, T., \& Pawlowski, J. (2018). Supervised machine learning outperforms taxonomy-based environmental DNA metabarcoding applied to biomonitoring. Molecular Ecology Resources, 18(6), 1381-1391. https://doi.org/10.1111/1755-0998.12926

Costa, C., Fanelli, E., Marini, S., Danovaro, R., \& Aguzzi, J. (2020). Global deep-sea biodiversity research trends highlighted by science mapping approach. Frontiers in Marine Science, 7, 384. https://doi. org/10.3389/fmars.2020.00384

Costello, M. J., \& Chaudhary, C. (2017). Marine biodiversity, biogeography, deep-sea gradients, and conservation. Current Biology, 27(11), R511-R527. https://doi.org/10.1016/j.cub.2017.04.060

Costello, M. J., Cheung, A., \& De Hauwere, N. (2010). Surface area and the seabed area, volume, depth, slope, and topographic variation for the world's seas, oceans, and countries. Environmental Science \& Technology, 44(23), 8821-8828. https://doi.org/10.1021/es1012752

Creer, S., Fonseca, V. G., Porazinska, D. L., Giblin-davis, R. M., Sung, W., Power, D. M., Packer, M., Carvalho, G. R., Blaxter, M. L., Lambshead, P. J. D., \& Thomas, W. K. (2010). Ultrasequencing of the meiofaunal biosphere: Practice, pitfalls and promises. Molecular Ecology, 19(Suppl 1), 4-20. https://doi.org/10.1111/j.1365-294X.2009.04473.x

Danovaro, R., Snelgrove, P. V., \& Tyler, P. (2014). Challenging the paradigms of deep-sea ecology. Trends in Ecology \& Evolution, 29(8), 465-475. https://doi.org/10.1016/j.tree.2014.06.002
Davis, N. M., Proctor, D. M., Holmes, S. P., Relman, D. A., \& Callahan, B. J. (2018). Simple statistical identification and removal of contaminant sequences in marker-gene and metagenomics data. Microbiome, 6(1), 226. https://doi.org/10.1186/s40168-018-0605-2

Deiner, K., Renshaw, M. A., Li, Y., Olds, B. P., Lodge, D. M., \& Pfrender, M. E. (2017). Long-range PCR allows sequencing of mitochondrial genomes from environmental DNA. Methods in Ecology and Evolution, 8(12), 1888-1898. https://doi.org/10.1111/2041-210X.12836

Dell'Anno, A., Carugati, L., Corinaldesi, C., Riccioni, G., \& Danovaro, R. (2015). Unveiling the biodiversity of deep-sea nematodes through metabarcoding: Are we ready to bypass the classical taxonomy? PLoS One, 10(12), e0144928. https://doi.org/10.1371/journ al.pone.0144928

Denonfoux, J., Parisot, N., Dugat-Bony, E., Biderre-Petit, C., Boucher, D., Morgavi, D. P., Le Paslier, D., Peyretaillade, E., \& Peyret, P. (2013). Gene capture coupled to high-throughput sequencing as a strategy for targeted metagenome exploration. DNA Research, 20(2), 185196. https://doi.org/10.1093/dnares/dst001

Derocles, S. A. P., Bohan, D. A., Dumbrell, A. J., Kitson, J. J. N., Massol, F., Pauvert, C., \& Evans, D. M. (2018). Biomonitoring for the 21st century: Integrating next-generation sequencing into ecological network analysis. In D. A. Bohan, A. J. Dumbrell, G. Woodward, \& M. Jackson (Eds.), Advances in ecological research, Vol. 58 (pp. 1-62). Academic Press.

Dugat-Bony, E., Peyretaillade, E., Parisot, N., Biderre-Petit, C., Jaziri, F., Hill, D., Rimour, S., \& Peyret, P. (2012). Detecting unknown sequences with DNA microarrays: explorative probe design strategies. Environmental Microbiology, 14(2), 356-371. https://doi. org/10.1111/j.1462-2920.2011.02559.x

Förster, D. W., Bull, J. K., Lenz, D., Autenrieth, M., Paijmans, J. L. A., Kraus, R. H. S., Nowak, C., Bayerl, H., Kuehn, R., Saveljev, A. P., Sindičić, M., Hofreiter, M., Schmidt, K., \& Fickel, J. (2018). Targeted resequencing of coding DNA sequences for SNP discovery in nonmodel species. Molecular Ecology Resources, 18(6), 1356-1373. https://doi. org/10.1111/1755-0998.12924

Frøslev, T. G., Kjøller, R., Bruun, H. H., Ejrnæs, R., Brunbjerg, A. K., Pietroni, C., \& Hansen, A. J. (2017). Algorithm for post-clustering curation of DNA amplicon data yields reliable biodiversity estimates. Nature Communications, 8(1), 1-11. https://doi.org/10.1038/ s41467-017-01312-x

Gambi, C., \& Danovaro, R. (2016). Biodiversity and life strategies of deep-sea meiofauna and nematode assemblages in the Whittard Canyon (Celtic margin, NE Atlantic Ocean). Deep Sea Research Part I: Oceanographic Research Papers, 108, 13-22. https://doi. org/10.1016/j.dsr.2015.12.001

Gasc, C., \& Peyret, P. (2017). Revealing large metagenomic regions through long DNA fragment hybridization capture. Microbiome, 5(1), 33. https://doi.org/10.1186/s40168-017-0251-0

Gasc, C., \& Peyret, P. (2018). Hybridization capture reveals microbial diversity missed using current profiling methods. Microbiome, 6(1), 61. https://doi.org/10.1186/s40168-018-0442-3

Gasc, C., Peyretaillade, E., \& Peyret, P. (2016). Sequence capture by hybridization to explore modern and ancient genomic diversity in model and nonmodel organisms. Nucleic Acids Research, 44(10), 4504-4518. https://doi.org/10.1093/nar/gkw309

Gauthier, M., Konecny-Dupré, L., Nguyen, A., Elbrecht, V., Datry, T., Douady, C., \& Lefébure, T. (2020). Enhancing DNA metabarcoding performance and applicability with bait capture enrichment and DNA from conservative ethanol. Molecular Ecology Resources, 20(1), 79-96. https://doi.org/10.1111/1755-0998.13088

Ghurye, J. S., Cepeda-Espinoza, V., \& Pop, M. (2016). Metagenomic assembly: Overview, challenges and applications. Yale Journal of Biology and Medicine, 89(3), 353-362.

Giebner, H., Langen, K., Bourlat, S. J., Kukowka, S., Mayer, C., Astrin, J. J., Misof, B., \& Fonseca, V. G. (2020). Comparing diversity levels in environmental samples: DNA sequence capture and metabarcoding 
approaches using $18 \mathrm{~S}$ and COI genes. Molecular Ecology Resources, 20(5), 1333-1345. https://doi.org/10.1111/1755-0998.13201

Gnirke, A., Melnikov, A., Maguire, J., Rogov, P., LeProust, E. M., Brockman, W., Fennell, T., Giannoukos, G., Fisher, S., Russ, C., Gabriel, S., Jaffe, D. B., Lander, E. S., \& Nusbaum, C. (2009). Solution hybrid selection with ultra-long oligonucleotides for massively parallel targeted sequencing. Nature Biotechnology, 27(2), 182-189. https://doi. org/10.1038/nbt.1523

Hajibabaei, M., Shokralla, S., Zhou, X., Singer, G. A., \& Baird, D. J. (2011). Environmental barcoding: A next-generation sequencing approach for biomonitoring applications using river benthos. PLoS One, 6(4), e17497. https://doi.org/10.1371/journal.pone.0017497

Jaziri, F., Parisot, N., Abid, A., Denonfoux, J., Ribiere, C., Gasc, C., Boucher, D., Brugere, J.-F., Mahul, A., Hill, D. R. C., Peyretaillade, E., \& Peyret, P. (2014). PhylOPDb: A 16S rRNA oligonucleotide probe database for prokaryotic identification. Database (Oxford), 2014, bau036. https://doi.org/10.1093/database/bau036

Kanagawa, T. (2003). Bias and artifacts in multitemplate polymerase chain reactions (PCR). Journal of Bioscience and Bioengineering, 96(4), 317-323. https://doi.org/10.1016/s1389-1723(03)90130-7

Katoh, K., Misawa, K., Kuma, K., \& Miyata, T. (2002). MAFFT: A novel method for rapid multiple sequence alignment based on fast Fourier transform. Nucleic Acids Research, 30(14), 3059-3066. https://doi. org/10.1093/nar/gkf436

Kawahara, A. Y., Breinholt, J. W., Espeland, M., Storer, C., Plotkin, D., Dexter, K. M., Toussaint, E. F. A., St Laurent, R. A., Brehm, G., Vargas, S., Forero, D., Pierce, N. E., \& Lohman, D. J. (2018). Phylogenetics of moth-like butterflies (Papilionoidea: Hedylidae) based on a new 13locus target capture probe set. Molecular Phylogenetics and Evolution, 127, 600-605. https://doi.org/10.1016/j.ympev.2018.06.002

Kopylova, E., Noé, L., \& Touzet, H. (2012). SortMeRNA: Fast and accurate filtering of ribosomal RNAs in metatranscriptomic data. Bioinformatics, 28(24), 3211-3217. https://doi.org/10.1093/bioin formatics/bts 611

Liu, S., Wang, X., Xie, L., Tan, M., Li, Z., Su, X. U., Zhang, H., Misof, B., Kjer, K. M., Tang, M., Niehuis, O., Jiang, H., \& Zhou, X. (2016). Mitochondrial capture enriches mito-DNA 100 fold, enabling PCR-free mitogenomics biodiversity analysis. Molecular Ecology Resources, 16(2), 470-479. https://doi.org/10.1111/1755-0998.12472

Machida, R. J., \& Knowlton, N. (2012). PCR primers for metazoan nuclear $18 \mathrm{~S}$ and $28 \mathrm{~S}$ ribosomal DNA sequences. PLoS One, 7(9), e46180. https://doi.org/10.1371/journal.pone.0046180

Mahé, F., Rognes, T., Quince, C., de Vargas, C., \& Dunthorn, M. (2015). Swarm v2: Highly-scalable and high-resolution amplicon clustering. PeerJ, 3, e1420. https://doi.org/10.7717/peerj.1420

Mamanova, L., Coffey, A. J., Scott, C. E., Kozarewa, I., Turner, E. H., Kumar, A., Howard, E., Shendure, J., \& Turner, D. J. (2010). Targetenrichment strategies for next-generation sequencing. Nature Methods, 7(2), 111-118. https://doi.org/10.1038/nmeth.1419

Martin, M. (2011). Cutadapt removes adapter sequences from highthroughput sequencing reads. Embnet Journal, 17(1), 10. https://doi. org/10.14806/ej.17.1.200

Mendoza, M. L. Z., Sicheritz-Pontén, T., \& Gilbert, M. T. P. (2014). Environmental genes and genomes: Understanding the differences and challenges in the approaches and software for their analyses. Briefings in Bioinformatics, 16(5), 745-758. https://doi.org/10.1093/ bib/bbv001

Mertes, F., ElSharawy, A., Sauer, S., van Helvoort, J. M. L. M., van der Zaag, P. J., Franke, A., Nilsson, M., Lehrach, H., \& Brookes, A. J. (2011). Targeted enrichment of genomic DNA regions for nextgeneration sequencing. Briefings in Functional Genomics, 10(6), 374386. https://doi.org/10.1093/bfgp/elr033

Meyer, M., \& Kircher, M. (2010). Illumina sequencing library preparation for highly multiplexed target capture and sequencing. Cold Spring Harbor Protocols, 2010(6), 1-10. https://doi.org/10.1101/pdb. prot5448
Militon, C., Rimour, S., Missaoui, M., Biderre, C., Barra, V., Hill, D., Moné, A., Gagne, G., Meier, H., Peyretaillade, E., \& Peyret, P. (2007). PhylArray: Phylogenetic probe design algorithm for microarray. Bioinformatics, 23(19), 2550-2557. https://doi.org/10.1093/bioin formatics/btm392

Miller, C. S., Baker, B. J., Thomas, B. C., Singer, S. W., \& Banfield, J. F. (2011). EMIRGE: Reconstruction of full-length ribosomal genes from microbial community short read sequencing data. Genome Biology, 12(5), R44. https://doi.org/10.1186/gb-2011-12-5-r44

Miya, M., Sato, Y., Fukunaga, T., Sado, T., Poulsen, J. Y., Sato, K., Minamoto, T., Yamamoto, S., Yamanaka, H., Araki, H., Kondoh, M., \& Iwasaki, W. (2015). MiFish, a set of universal PCR primers for metabarcoding environmental DNA from fishes: Detection of more than 230 subtropical marine species. Royal Society Open Science, 2(7), 150088. https://doi.org/10.1098/rsos.150088

Oksanen, J., Kindt, R., Legendre, P., O'Hara, B., Simpson, G. L., Solymos, P. M., \& Wagner, H. (2008). The vegan package. Community Ecology Package.

Parada, A. E., Needham, D. M., \& Fuhrman, J. A. (2016). Every base matters: Assessing small subunit rRNA primers for marine microbiomes with mock communities, time series and global field samples. Environmental Microbiology, 18(5), 1403-1414. https://doi. org/10.1111/1462-2920.13023

Parisot, N., Denonfoux, J., Dugat-Bony, E., Peyret, P., \& Peyretaillade, E. (2012). KASpOD-A web service for highly specific and explorative oligonucleotide design. Bioinformatics, 28(23), 3161-3162. https:// doi.org/10.1093/bioinformatics/bts597

Pericard, P., Dufresne, Y., Couderc, L., Blanquart, S., \& Touzet, H. (2018). MATAM: reconstruction of phylogenetic marker genes from short sequencing reads in metagenomes. Bioinformatics, 34(4), 585-591. https://doi.org/10.1093/bioinformatics/btx644

Porter, T. M., \& Hajibabaei, M. (2018). Scaling up: A guide to highthroughput genomic approaches for biodiversity analysis. Molecular Ecology, 27(2), 313-338. https://doi.org/10.1111/mec.14478

Pruesse, E., Quast, C., Knittel, K., Fuchs, B. M., Ludwig, W., Peplies, J., \& Glöckner, F. O. (2007). SILVA: A comprehensive online resource for quality checked and aligned ribosomal RNA sequence data compatible with ARB. Nucleic Acids Research, 35(21), 7188-7196. https:// doi.org/10.1093/nar/gkm864

Quast, C., Pruesse, E., Yilmaz, P., Gerken, J., Schweer, T., Yarza, P., Peplies, J., \& Glöckner, F. O. (2013). The SILVA ribosomal RNA gene database project: Improved data processing and web-based tools. Nucleic Acids Research, 41(Database issue), D590-D596. https:// doi.org/10.1093/nar/gks1219

Quince, C., Walker, A. W., Simpson, J. T., Loman, N. J., \& Segata, N. (2017). Erratum: Corrigendum: Shotgun metagenomics, from sampling to analysis. Nature Biotechnology, 35(12), 1211. https://doi. org/10.1038/nbt1217-1211b

Ranchou-Peyruse, M., Gasc, C., Guignard, M., Aüllo, T., Dequidt, D., Peyret, P., \& Ranchou-Peyruse, A. (2017). The sequence capture by hybridization: A new approach for revealing the potential of mono-aromatic hydrocarbons bioattenuation in a deep oligotrophic aquifer. Microbial Biotechnology, 10(2), 469-479. https://doi. org/10.1111/1751-7915.12426

Ribière, C., Beugnot, R., Parisot, N., Gasc, C., Defois, C., Denonfoux, J., \& Peyret, P. (2016). Targeted gene capture by hybridization to illuminate ecosystem functioning. In F. Martin, \& S. Uroz (Eds.), Microbial environmental genomics (MEG) (pp. 167-182). Springer.

Sayers, E. W., Cavanaugh, M., Clark, K., Ostell, J., Pruitt, K. D., \& KarschMizrachi, I. (2020). GenBank. Nucleic Acids Research, 48(D1), D84D86. https://doi.org/10.1093/nar/gkz956

Schmieder, R., \& Edwards, R. (2011). Quality control and preprocessing of metagenomic datasets. Bioinformatics, 27(6), 863-864. https:// doi.org/10.1093/bioinformatics/btr026

Schnell, I. B., Bohmann, K., \& Gilbert, M. T. P. (2015). Tag jumps illuminated - reducing sequence-to-sample misidentifications in 
metabarcoding studies. Molecular Ecology Resources, 15(6), 12891303. https://doi.org/10.1111/1755-0998.12402

Seeber, P. A., McEwen, G. K., Löber, U., Förster, D. W., East, M. L., Melzheimer, J., \& Greenwood, A. D. (2019). Terrestrial mammal surveillance using hybridization capture of environmental DNA from African waterholes. Molecular Ecology Resources, 19(6), 1486-1496. https://doi.org/10.1111/1755-0998.13069

Sefc, K. M., Payne, R. B., \& Sorenson, M. D. (2007). Single base errors in PCR products from avian museum specimens and their effect on estimates of historical genetic diversity. Conservation Genetics, 8(4), 879-884. https://doi.org/10.1007/s10592-006-9240-8

Shokralla, S., Gibson, J. F., King, I., Baird, D. J., Janzen, D. H., Hallwachs, W., \& Hajibabaei, M. (2016). Environmental DNA Barcode Sequence Capture: Targeted, PCR-free Sequence Capture for Biodiversity Analysis from Bulk Environmental Samples. bioRxiv, 087437. https://doi.org/10.1101/087437

Singer, G. A. C., Fahner, N. A., Barnes, J. G., McCarthy, A., \& Hajibabaei, M. (2019). Comprehensive biodiversity analysis via ultra-deep patterned flow cell technology: A case study of eDNA metabarcoding seawater. Scientific Reports, 9(1), 5991. https://doi.org/10.1038/ s41598-019-42455-9

Sinniger, F., Pawlowski, J., Harii, S., Gooday, A. J., Yamamoto, H., Chevaldonné, P., Cedhagen, T., Carvalho, G., \& Creer, S. (2016). Worldwide analysis of sedimentary DNA reveals major gaps in taxonomic knowledge of deep-sea benthos. Frontiers in Marine Science, 3, 92. https://doi.org/10.3389/fmars.2016.00092

Smyth, R. P., Schlub, T. E., Grimm, A., Venturi, V., Chopra, A., Mallal, S., Davenport, M. P., \& Mak, J. (2010). Reducing chimera formation during PCR amplification to ensure accurate genotyping. Gene, 469(1-2), 45-51. https://doi.org/10.1016/j.gene.2010.08.009

Taberlet, P., Coissac, E., Hajibabaei, M., \& Rieseberg, L. H. (2012). Environmental DNA. Molecular Ecology, 21(8), 1789-1793. https:// doi.org/10.1111/j.1365-294x.2012.05542.x

Thomsen, P. F., \& Willerslev, E. (2015). Environmental DNA - An emerging tool in conservation for monitoring past and present biodiversity. Biological Conservation, 183, 4-18. https://doi.org/10.1016/j. biocon.2014.11.019

Torti, A., Lever, M. A., \& Jørgensen, B. B. (2015). Origin, dynamics, and implications of extracellular DNA pools in marine sediments. Marine Genomics, 24, 185-196. https://doi.org/10.1016/j. margen.2015.08.007

Valentini, A., Pompanon, F., \& Taberlet, P. (2009). DNA barcoding for ecologists. Trends in Ecology \& Evolution, 24(2), 110-117. https://doi. org/10.1016/j.tree.2008.09.011

Wangensteen, O. S., Palacín, C., Guardiola, M., \& Turon, X. (2018). DNA metabarcoding of littoral hard-bottom communities: High diversity and database gaps revealed by two molecular markers. PeerJ, 6, e4705. https://doi.org/10.7717/peerj.4705
Wilcox, T. M., Zarn, K. E., Piggott, M. P., Young, M. K., McKelvey, K. S., \& Schwartz, M. K. (2018). Capture enrichment of aquatic environmental DNA: A first proof of concept. Molecular Ecology Resources, 18(6), 1392-1401. https://doi. org/10.1111/1755-0998.12928

Wilson, J. J., Brandon-Mong, G. J., Gan, H. M., \& Sing, K. W. (2018). Highthroughput terrestrial biodiversity assessments: Mitochondrial metabarcoding, metagenomics or metatranscriptomics? Mitochondrial DNA Part A: DNA Mapping, Sequencing, and Analysis, 30(1), 60-67. https://doi.org/10.1080/24701394.2018.1455189

Wood, D. E., Lu, J., \& Langmead, B. (2019). Improved metagenomic analysis with Kraken 2. Genome Biology, 20(1), 257. https://doi. org/10.1186/s13059-019-1891-0

Wood, D. E., \& Salzberg, S. L. (2014). Kraken: Ultrafast metagenomic sequence classification using exact alignments. Genome Biology, 15(3), R46. https://doi.org/10.1186/gb-2014-15-3-r46

$\mathrm{Xu}, \mathrm{J.}$ (2006). Microbial ecology in the age of genomics and metagenomics: Concepts, tools, and recent advances. Molecular Ecology, 15(7), 1713-1731. https://doi.org/10.1111/j.1365-294x.2006.02882.x

Yamamoto, S., Masuda, R., Sato, Y., Sado, T., Araki, H., Kondoh, M., Minamoto, T., \& Miya, M. (2017). Environmental DNA metabarcoding reveals local fish communities in a species-rich coastal sea. Scientific Reports, 7, 40368. https://doi.org/10.1038/srep4 0368

Zeppilli, D., Leduc, D., Fontanier, C., Fontaneto, D., Fuchs, S., Gooday, A. J., Goineau, A., Ingels, J., Ivanenko, V. N., Kristensen, R. M., Neves, R. C., Sanchez, N., Sandulli, R., Sarrazin, J., Sørensen, M. V., Tasiemski, A., Vanreusel, A., Autret, M., Bourdonnay, L., ... Fernandes, D. (2018). Characteristics of meiofauna in extreme marine ecosystems: A review. Marine Biodiversity, 48(1), 35-71. https://doi.org/10.1007/s12526-017-0815-z

\section{SUPPORTING INFORMATION}

Additional supporting information may be found in the online version of the article at the publisher's website.

How to cite this article: Günther, B., Marre, S., Defois, C., Merzi, T., Blanc, P., Peyret, P., \& Arnaud-Haond, S. (2022). Capture by hybridization for full-length barcode-based eukaryotic and prokaryotic biodiversity inventories of deep sea ecosystems. Molecular Ecology Resources, 22, 623-637. https://doi.org/10.1111/1755-0998.13500 